\title{
Extending the Impact of RAC1b Overexpression to Follicular Thyroid Carcinomas
}

\author{
Márcia Faria, ${ }^{1}$ Liliana Capinha, ${ }^{1}$ Joana Simões-Pereira, ${ }^{2}$ \\ Maria João Bugalho, ${ }^{1,2,3}$ and Ana Luísa Silva ${ }^{1}$ \\ ${ }^{1}$ Unidade de Investigação de Patobiologia Molecular, Instituto Português de Oncologia de Lisboa Francisco Gentil EPE, \\ 1099-023 Lisboa, Portugal \\ ${ }^{2}$ Serviço de Endocrinologia, Instituto Português de Oncologia de Lisboa Francisco Gentil EPE, 1099-023 Lisboa, Portugal \\ ${ }^{3}$ Clínica Universitária de Endocrinologia, NOVA Medical School/Faculdade de Ciências Médicas, \\ Universidade Nova de Lisboa, 1169-056 Lisboa, Portugal
}

Correspondence should be addressed to Ana Luísa Silva; silva.r.analuisa@gmail.com

Received 14 January 2016; Accepted 20 March 2016

Academic Editor: Carlo Cappelli

Copyright (C) 2016 Márcia Faria et al. This is an open access article distributed under the Creative Commons Attribution License, which permits unrestricted use, distribution, and reproduction in any medium, provided the original work is properly cited.

RAClb is a hyperactive variant of the small GTPase RAC1 known to be a relevant molecular player in different cancers. Previous studies from our group lead to the evidence that its overexpression in papillary thyroid carcinoma (PTC) is associated with an unfavorable prognosis. In the present study, we intended to extend the analysis of RAClb expression to thyroid follicular neoplasms and to seek for clinical correlations. RAClb expression levels were determined by RT-qPCR in thyroid follicular tumor samples comprising 23 follicular thyroid carcinomas (FTCs) and 33 follicular thyroid adenomas (FTAs). RAClb was found to be overexpressed in 33\% of carcinomas while no RAClb overexpression was documented among follicular adenomas. Patients with a diagnosis of FTC were divided into two groups based on longitudinal evolution and final outcome. RAClb overexpression was significantly associated with both the presence of distant metastases $(P=0.01)$ and poorer clinical outcome $(P=0.01)$ suggesting that, similarly to that previously found in PTCs, RAClb overexpression in FTCs is also associated with worse outcomes. Furthermore, the absence of RAClb overexpression in follicular adenomas hints its potential as a molecular marker likely to contribute, in conjunction with other putative markers, to the preoperative differential diagnosis of thyroid follicular lesions.

\section{Introduction}

More than $95 \%$ of thyroid cancers originate from thyroid follicular epithelial cells. Among the well-differentiated forms, papillary thyroid carcinoma (PTC) is the most prevalent one, accounting for $75-80 \%$ of cases, followed by the follicular thyroid carcinoma (FTC), which represents approximately $10-15 \%$ of all thyroid cancers [1]. Distinction between FTC and its benign counterpart (follicular adenoma) is impossible on cytological grounds. Pathological examination showing capsular or vascular invasion is necessary to establish the diagnosis of FTC [2-4]. Activating alterations in the canonical Ras/Raf/MEK/ERK pathway (MAPK pathway) are considered to have key role in thyroid carcinogenesis $[1,5]$. The BRAF V600E activating mutation is the most frequent genetic alteration in PTCs. In FTCs, however, this alteration is virtually absent and oncogenic alterations of RAS proteins are instead the most prevalent ones $[1,5]$. Although a single oncogenic alteration in MAPK pathway might be sufficient to drive thyroid cell neoplastic transformation, further supportive molecular events are likely to be associated with thyroid malignant progression leading to more aggressive phenotypes and poorer clinical outcomes. We have recently described RAClb, a splicing variant of RACl, as a potential new prognostic marker for clinical outcome in PTC patients [6].

RACl belongs to the Rho family of Ras-like small GTPbinding proteins, a class of molecular "switches" that regulate cellular functions by cycling between an inactive, GDPand an active, GTP-bound state [7]. These small GTPases have been implicated in cancer since they regulate signaling pathways involved in processes such as gene expression, 
cell proliferation, and cell migration [8]. The RAC1 splice variant, RAClb, contains 57 additional nucleotides that result in an in-frame insertion of 19 amino acid residues in the vicinity of an important regulatory region of the GTPase [9]. This confers RAClb activating properties and a selective downstream signaling in comparison to RAC1 [10].

$\mathrm{RAClb}$ overexpression has been documented in colorectal, breast, lung, and pancreatic cancer [9, 11-15]. In our previous study, we reported for the first time RAClb expression in thyroid tissue [6]. We have, previously, shown that RAClb is overexpressed in PTCs compared to normal thyroid tissue and that RAClb overexpression is significantly associated with BRAF V600E mutation and poorer clinical outcome in PTC [6]. Here, we aimed to broaden the study of RAClb expression to follicular lesions including follicular adenomas and follicular carcinomas and to seek for clinical correlations.

\section{Materials and Methods}

2.1. Tumor Samples. Samples representative of 23 FTCs and samples representative of 33 follicular adenomas from 56 patients, who underwent surgery at our institution, were analyzed. Samples were collected at surgery and immediately frozen and stored in liquid nitrogen. Tissue sample collection was carried out in accordance with protocols approved by the institutional review board and written informed consent was obtained together with the consent for surgery.

2.2. RNA Extraction, cDNA Synthesis, and Mutational Screening of KRAS, HRAS, and NRAS. Total RNA was obtained from frozen tissues using RNA easykit (Qiagen), according to manufacturer's instructions, and $2 \mu \mathrm{g}$ was reverse transcribed using random primers and SuperScript II (Invitrogen).

Mutational analysis of KRAS, HRAS, and NRAS was performed by Sanger sequencing method: the full coding regions of $R A S$ transcripts were amplified by reverse transcription polymerase chain reaction (RT-PCR) (primers and PCR conditions available upon request). PCR purified products were directly sequenced using BigDye ${ }^{\circledR}$ Terminator vl.1 Cycle Sequencing Kit (Applied Biosystems, Foster City, CA, USA). Sequencing products were analyzed using ABI PRISM 3130 Genetic Analyzer (Applied Biosystems).

2.3. RT-qPCR. The RAClb and total RAC1 expression levels were quantified by quantitative reverse transcription polymerase chain reaction (RT-qPCR) on an ABI Prism 7900HT Sequence Detection System, as previously described [6]. For each sample, RAClb levels were normalized to total RAC1 $(\mathrm{RAClb}+\mathrm{RACl})$ expression level. RAClb normalized values were then expressed relative to that of a pool of normal thyroid tissues, used as reference sample. Expression values correspond to arbitrary units representing fold differences relative to the reference sample. RAClb overexpression was defined as a value above a threshold level of RAClb expression corresponding to the mean plus two standard deviations of the RAClb expression level found in the set of normal thyroid tissue samples; this threshold level was set at 2.133 (arbitrary units). Similarly, to monitor total RAC1 expression among samples, total Racl levels were normalized to betaactin expression level (housekeeping gene normalization) and expressed relative to that of the reference sample.

2.4. Protein Lysates and Western Blotting. Total protein lysates were prepared from frozen thyroid tissues as previously described [6]. The primary antibodies rabbit polyclonal anti-Raclb (Millipore) and mouse monoclonal anti- $\beta$-actin (Sigma) were used in Western blot at 1:1000 and 1:3000 dilutions, respectively. Detection was carried out using secondary peroxidase-conjugated anti-mouse IgG (Bio-Rad) or anti-rabbit IgG (Bio-Rad) antibodies followed by chemiluminescence.

2.5. Statistical Analysis. Statistical analysis was carried out using GraphPad Prism statistical software (San Diego, CA). When appropriate, values are expressed as mean \pm SD. Statistical comparisons of rates and proportions were made using unpaired two-tailed Student's $t$-test or the two-tailed Fisher exact test, when appropriated. Statistical significance was accepted at $P<0.05$.

\section{Results}

3.1. RAClb Is Overexpressed in FTC. Total RACl and RAClb expression levels were assessed by RT-qPCR. No significant differences in total RACl levels were found between FTCs and FTAs samples. RAClb expression levels in tumor samples were obtained following normalization to the levels from a pool of normal thyroid tissues used as reference sample in all RT-qPCR assays. The mean level for RAClb expression was significantly higher in FTCs $(2.137 \pm 0.5515)$ compared to FTAs $(0.9656 \pm 0.07342)$ ( $P$ value $=0.0152$, two-tailed Student's $t$-test), corresponding to a 2.2 mean fold increase in the levels of RAClb expression (Figure 1(a)).

In order to distinguish tumors that overexpressed $\mathrm{RAClb}$ from those that did not, we defined a threshold level of $\mathrm{RAClb}$ expression relative to the normal thyroid tissue (see Section 2) above where we considered RAClb to be overexpressed. RAClb was found to be overexpressed in 7 out of 23 FTCs (30\%). Notably, none of the cases belonging to the FTAs group was found to overexpress RAClb. Consistently, the difference in RAClb overexpression between follicular adenomas and carcinomas was statistically significant $(P$ value $=0.001$, two-tailed Fisher's exact test; Figure 1(b)) .

Since the assessment of RAClb overexpression by immunohistochemistry in paraffin-embedded tissues might be relevant for diagnostic purposes, we further explored this possibility using the only RAClb specific antibody that is commercially available. Unfortunately, we were not able to detect a clear difference in RAClb protein levels either comparing $\mathrm{RAClb}$ overexpressing and nonoverexpressing tumors or tumor and normal tissue. Nevertheless, a notorious difference in $\mathrm{RAClb}$ expression was observed when protein levels were assessed by Western blot. This discrepancy is likely to be due to antibody nonspecific binding as pointed out by the band of $\cong 50 \mathrm{kDa}$ observed in the immunoblot 


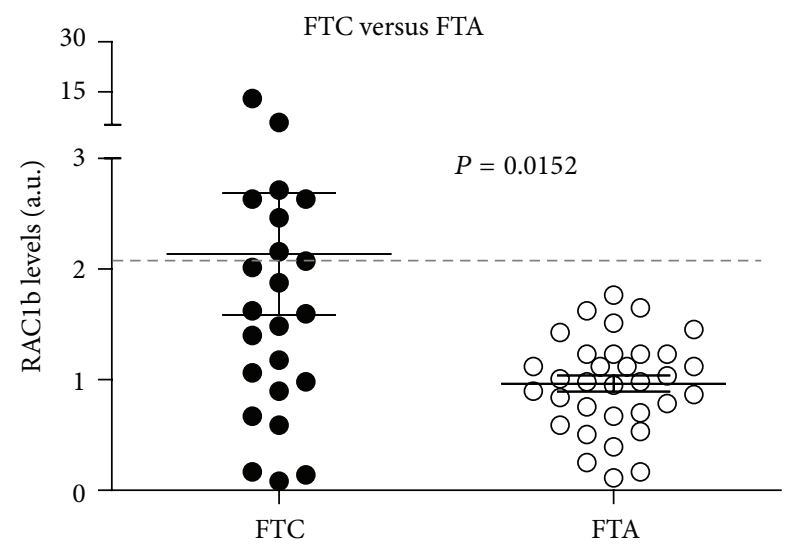

FTC

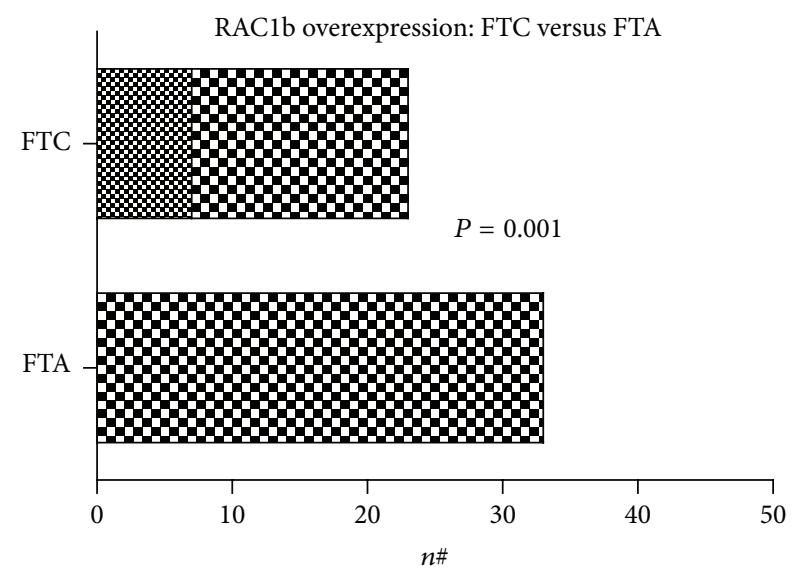

\% RAClb+

QD RAClb-

(a)

(b)

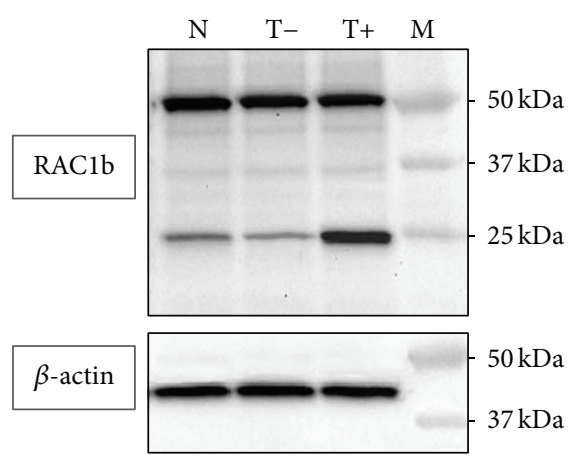

(c)

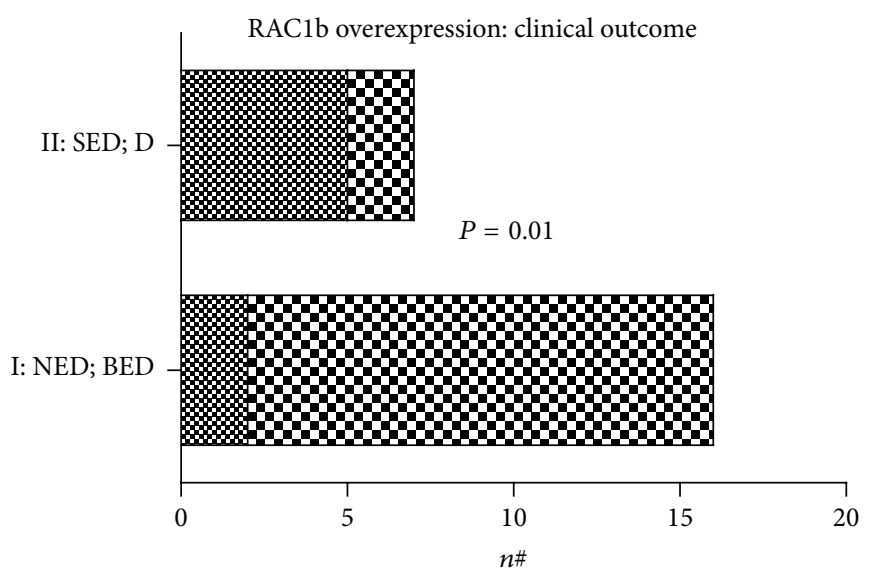

RAClb+ Da RAClb-



$\%$ RAClb+ DaClb-

(d)

(e)

Figure 1: RAClb expression in follicular thyroid tumors. (a) Expression levels of RAClb among FTCs $(n=23)$ and FTAs $(n=33)$. RAC1b expression levels, quantified by qRT-PCR, correspond to arbitrary units representing fold differences relative to the reference sample; the threshold value defining RAClb overexpression was set at 2.133 (corresponding to the mean plus two standard deviations of RAClb expression level in a set of normal thyroid tissue samples). (b) Comparative analysis of RAClb overexpression (RAClb+) between follicular thyroid carcinomas (FTC) and follicular thyroid adenomas (FTA). (c) Western blot analysis of RAClb protein levels in normal thyroid (N) and RAClb overexpressing $(\mathrm{T}+)$ and nonoverexpressing $(\mathrm{T}-)$ tumors; protein molecular weight marker $(\mathrm{M})$. (d) Association of RAClb overexpression $(\mathrm{RAClb}+)$ with clinical outcome (Group I: NED: no evidence of disease, BED: biochemical evidence of disease; Group II: SED: structural evidence of disease, D: death due to disease). (e) Association of RAClb overexpression (RAClb+) with the presence (M1) or absence (M0) of distant metastases. 
(Figure 1(c)) that hampers an accurate analysis by immunohistochemistry.

3.2. RAC1b Overexpression Is Associated with Presence of Distant Metastases and Poorer Clinical Outcomes. To investigate whether RAClb overexpression was associated with histopathological parameters and clinical outcome, FTCs were grouped based on the clinical data available (see Table 1).

A comparative analysis between FTC patients without distant metastases (M0, 16 cases) versus FTC patients with distant metastases (M1, 7 cases) disclosed a higher prevalence of RAClb overexpression in the latter group (M1-5/7; M0$2 / 16)$. The association between RAClb overexpression and presence of distant metastases was statistically significant $(P$ value $=0.01$; Figure $1(\mathrm{c})$ ). No statistical significant correlation was found between RAClb overexpression and histopathological features such as multifocality, angioinvasion, Hürthle cell subtype, or presence of poorly differentiated areas.

FTCs were grouped based on the final clinical outcome. Group I (70\% of patients) included patients who reached full sustained remission (no evidence of disease) and patients presenting only biochemical evidence of disease. In contrast, Group II (30\% of patients) included patients with structural evidence of disease and those who died due to disease progression. The correlation between RAClb overexpression and Group II was statistically significant $(P$ value $=0.01$; Figure 1(d)), reinforcing that RAClb might be associated with poorer clinical outcomes in FTC patients.

\section{Discussion}

At present, stratification risk of patients with follicular cellderived thyroid carcinomas mainly relies on clinical and histological criteria proved to be insufficient to tailor case management to individual risk levels [16]. A major effort to improve the panel of prognostic indicators has entailed the molecular characterization of thyroid tumors, seeking for molecular markers with a reliable prognostic value $[17,18]$.

Previous studies from our group provided evidence for a contributory role of RAClb in PTC development and clinical outcome [6]. We, therefore, hypothesized that RAClb might also be involved in follicular thyroid carcinoma development. Moreover, taking into account the impossibility of distinguishing a follicular carcinoma from a follicular adenoma, preoperatively, we wondered whether the RAClb expression could contribute to the distinction of these two entities.

Our data suggest that RAClb is likely to have a differential expression in follicular adenomas and carcinomas, since RAClb was overexpressed in $30 \%$ of FTCs whereas no overexpression was found among FTAs. Furthermore, we also found a significant correlation between RAClb overexpression and the presence of distant metastases, hence, with poorer outcome.

Papillary thyroid carcinoma and follicular thyroid carcinoma are collectively designated well-differentiated thyroid carcinomas (DTCs). Nonetheless, the underlying genetic alterations promoting the development of these two types of thyroid cancer are different. The BRAF V600E mutation occurs in approximately $45 \%$ of PTCs. It is not definitely established whether BRAF V600E initiates PTC tumorigenesis or is a secondary event. In a previous study, we have shown that RAClb overexpression significantly associates with BRAF V600E mutation in PTCs [10]. This suggests that a functional cooperation between RAClb and BRAF V600E may play a role in PTC progression, similarly to that previously described in colorectal cancer [11]. In fact, in colorectal tumors, the elevated levels of Raclb expression were significantly associated with mutant BRAF but not with mutant K-RAS genotype, suggesting that RAClb is needed to sustain BRAF-induced but not RAS-induced cell transformation. The mechanism of action of RAClb in tumorigenesis may, however, differ in other tissues.

Concerning follicular thyroid lesions, BRAF V600E is virtually absent in this tumor subtype and the most common MAPK-pathway alteration is the presence of RAS mutations. Notably and contrary to that found in colorectal cancer, oncogenic alterations in K-RAS have also been associated with RAClb overexpression in lung cancer and RAClb was shown to promote K-RAS-induced lung tumorigenesis [13]. In our series of FTC patients no association between RAClb overexpression and RAS mutations could be observed. However, this might be due to the small sample size. Despite these, the incidence of RAS mutations in follicular adenomas, which are considered premalignant lesions, suggests a role for RAS in early phases of follicular cell tumorigenesis. This is in agreement with the hypothesis that the progression of a premalignant lesion into cancer, with different grades of aggressiveness, is linked to a continuum of molecular changes dependent on late and/or modifier events that are not necessarily exclusive of a specific type of tumor or mechanism of tumorigenesis. Overexpression of RAClb may thus be one of these modifier events that can occur in different subtypes of DTCs despite having different triggers.

\section{Conclusion}

The clinical usefulness of RAClb overexpression in the context of thyroid malignancies remains to be definitively established. Nevertheless, our data suggest that RAClb might be involved in the modulation of malignant progression of FTCs, contributing to poorer clinical outcomes, similarly to that previously documented in PTCs. Our data also support the potential of RAClb as a molecular marker likely to contribute, in conjunction with other putative markers, to the diagnosis and prognosis of FTCs.

\section{Competing Interests}

The authors declare that they have no competing interests.

\section{Acknowledgments}

This work was partially supported by the funding program of IPOLFG research projects, 2013, and by the Associação de Endocrinologia Oncologica. 







\section{References}

[1] F. Bhaijee and Y. E. Nikiforov, "Molecular analysis of thyroid tumors," Endocrine Pathology, vol. 22, no. 3, pp. 126-133, 2011.

[2] D. S. Cooper, G. M. Doherty, B. R. Haugen et al., "Management guidelines for patients with thyroid nodules and differentiated thyroid cancer," Thyroid, vol. 16, no. 2, pp. 109-141, 2006.

[3] B. R. Haugen, E. K. Alexander, K. C. Bible et al., "2015 American Thyroid Association Management Guidelines for Adult Patients with Thyroid Nodules and Differentiated Thyroid Cancer: the American Thyroid Association Guidelines Task Force on Thyroid Nodules and Differentiated Thyroid Cancer," Thyroid, vol. 26, no. 1, pp. 1-133, 2016.

[4] R. A. DeLellis, "Pathology and genetics of thyroid carcinoma," Journal of Surgical Oncology, vol. 94, no. 8, pp. 662-669, 2006.

[5] M. Romitti, L. Ceolin, D. R. Siqueira, C. V. Ferreira, S. M. Wajner, and A. L. Maia, "Signaling pathways in follicular cellderived thyroid carcinomas (review)," International Journal of Oncology, vol. 42, no. 1, pp. 19-28, 2013.

[6] A. L. Silva, F. Carmo, and M. J. Bugalho, "RAClb overexpression in papillary thyroid carcinoma: a role to unravel," European Journal of Endocrinology, vol. 168, no. 6, pp. 795-804, 2013.

[7] A. B. Jaffe and A. Hall, "Rho GTPases: biochemistry and biology," Annual Review of Cell and Developmental Biology, vol. 21, pp. 247-269, 2005.

[8] J. L. Orgaz, C. Herraiz, and V. Sanz-Moreno, "Rho GTPases modulate malignant transformation of tumor cells," Small GTPases, vol. 5, no. 4, Article ID e28209, 2014.

[9] P. Jordan, R. Brazão, M. G. Boavida, C. Gespach, and E. Chastre, "Cloning of a novel human Raclb splice variant with increased expression in colorectal tumors," Oncogene, vol. 18, no. 48, pp. 6835-6839, 1999.

[10] D. Fiegen, L.-C. Haeusler, L. Blumenstein et al., "Alternative Splicing of Racl Generates Raclb, a Self-activating GTPase," Journal of Biological Chemistry, vol. 279, no. 6, pp. 4743-4749, 2004.

[11] P. Matos, C. Oliveira, S. Velho et al., "B-Raf V600E cooperates with alternative spliced Raclb to sustain colorectal cancer cell survival," Gastroenterology, vol. 135, no. 3, pp. 899-906, 2008.

[12] A. Schnelzer, D. Prechtel, U. Knaus et al., "Racl in human breast cancer: overexpression, mutation analysis, and characterization of a new isoform, Raclb," Oncogene, vol. 19, no. 26, pp. 3013$3020,2000$.

[13] C. Zhou, S. Licciulli, J. L. Avila et al., "The Racl splice form Raclb promotes K-ras-induced lung tumorigenesis," Oncogene, vol. 32, no. 7, pp. 903-909, 2013.

[14] C. Mehner, E. Miller, D. Khauv et al., "Tumor cell-derived MMP3 orchestrates Raclb and tissue alterations that promote pancreatic adenocarcinoma," Molecular Cancer Research, vol. 12, no. 10, pp. 1430-1439, 2014.

[15] H. Ungefroren, S. Sebens, K. Giehl et al., "Raclb negatively regulates TGF- $\beta 1$-induced cell motility in pancreatic ductal epithelial cells by suppressing Smad signalling," Oncotarget, vol. 5, no. 1, pp. 277-290, 2014.

[16] J. A. Sipos and E. L. Mazzaferri, "Thyroid cancer epidemiology and prognostic variables," Clinical Oncology, vol. 22, no. 6, pp. 395-404, 2010.

[17] R. L. Witt, R. L. Ferris, E. A. Pribitkin, S. I. Sherman, D. L. Steward, and Y. E. Nikiforov, "Diagnosis and management of differentiated thyroid cancer using molecular biology," The Laryngoscope, vol. 123, no. 4, pp. 1059-1064, 2013.
[18] S. Nagar, S. Ahmed, C. Peeples et al., "Evaluation of genetic biomarkers for distinguishing benign from malignant thyroid neoplasms," American Journal of Surgery, vol. 207, no. 4, pp. 596-601, 2014. 


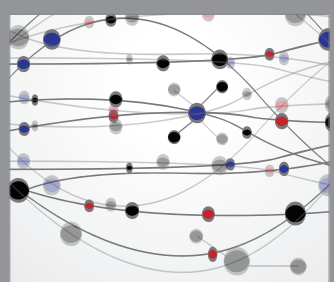

The Scientific World Journal
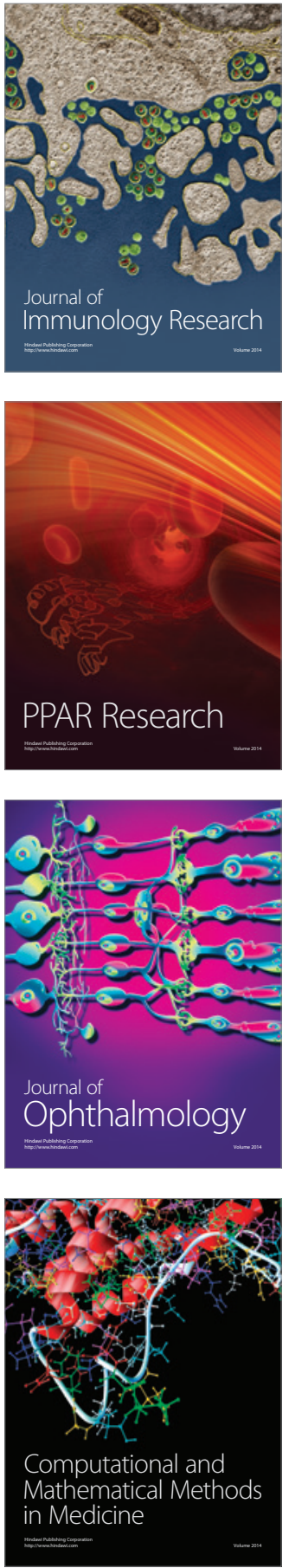

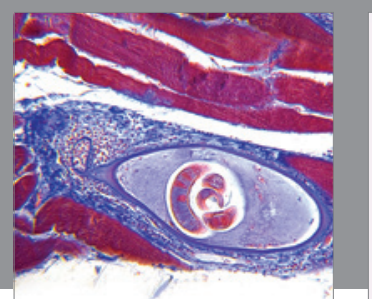

Gastroenterology Research and Practice

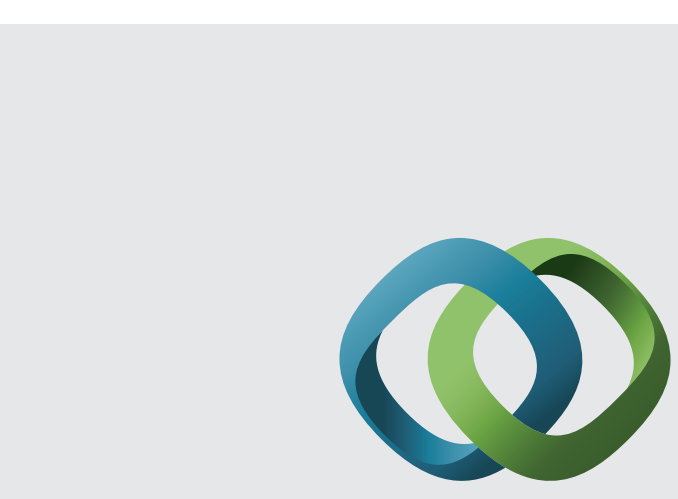

\section{Hindawi}

Submit your manuscripts at

http://www.hindawi.com
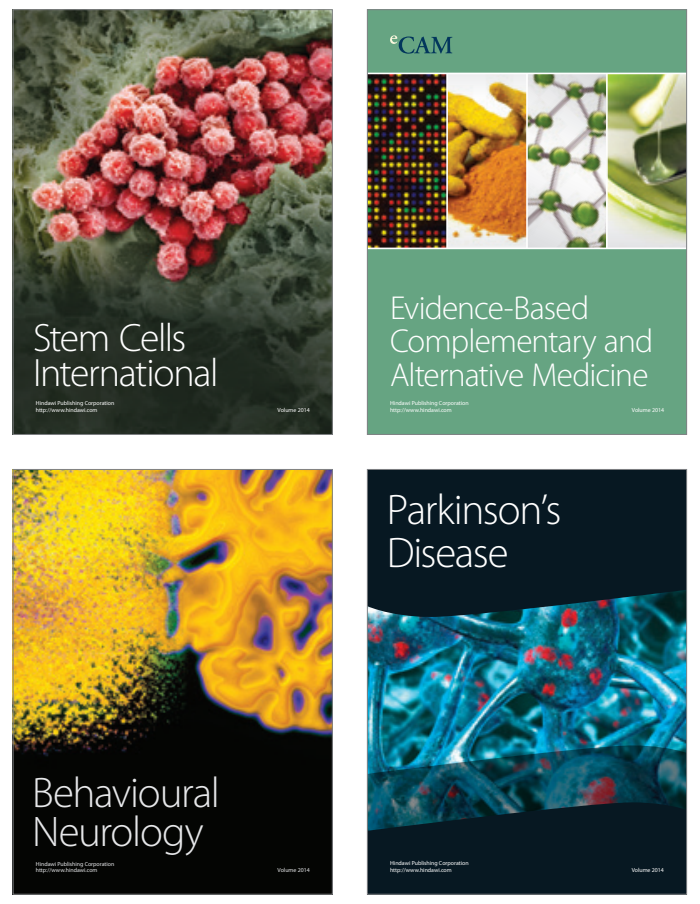
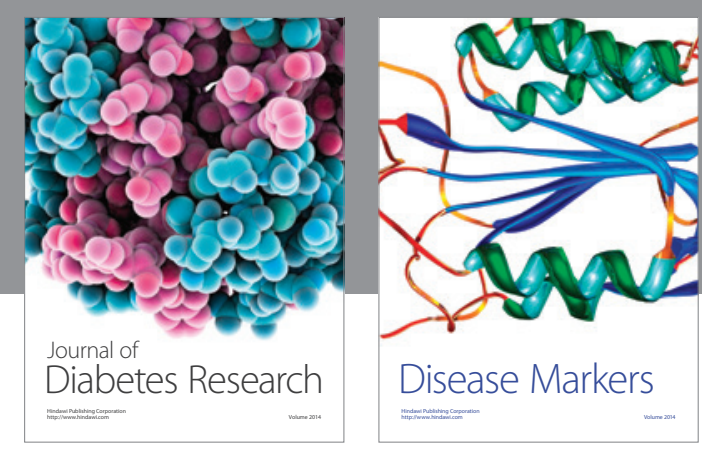

Disease Markers
